\title{
Endoscopic versus open microvascular decompression for trigeminal neuralgia: a systematic review and comparative meta-analysis
}

\author{
Nirmeen Zagzoog, MSc, MD, ${ }^{1}$ Ahmed Attar, MBBCh, FRCPC, ABPN, ${ }^{2}$ Radwan Takroni, MBBCh, ${ }^{1}$ \\ Mazen B. Alotaibi, MBBCh, ${ }^{1}$ and Kesh Reddy, MD, FRCSC, DABNS ${ }^{1}$
}

\begin{abstract}
'Division of Neurosurgery, Department of Surgery; and 2Division of Neurology, Department of Medicine, Hamilton General
\end{abstract} Hospital, Hamilton, Ontario, Canada

\begin{abstract}
OBJECTIVE Microvascular decompression (MVD) is commonly used in the treatment of trigeminal neuralgia (TN) with positive clinical outcomes. Fully endoscopic MVD (E-MVD) has been proposed as an effective minimally invasive alternative, but a comparative review of the two approaches has not been conducted. The authors performed a metaanalysis of studies, comparing patient outcome rates and complications for the open versus the endoscopic technique. METHODS The PubMed/MEDLINE and Ovid databases were searched for studies published from database inception to 2017. The search terms used included, but were not limited to, "open microvascular decompression," "microvascular decompression for trigeminal neuralgia," and "endoscopic decompression for trigeminal neuralgia." Criteria for inclusion of studies in the meta-analysis were established as follows: adult patients, clinical studies with $\geq 10$ patients (excluding case studies to obtain a higher volume of outcome rates), utilization of open MVD or E-MVD to treat TN, craniotomy and retrosigmoid incision, English-language studies, and articles that listed pain relief outcomes (complete, very good, partial, or absent), recurrence rate (number of patients), and complications (paresis, hearing loss, CSF leakage, cerebellar damage, infection, death). Relevant references from the chosen articles were also included.
\end{abstract}

RESULTS From a larger pool of 1039 studies, 23 articles were selected for review: 13 on traditional MVD and 10 on E-MVD. The total number of patients was 6749 , of which 5783 patients (and 5802 procedures) had undergone MVD and 993 patients (and procedures) had undergone E-MVD. Analyzed data included postoperative pain relief outcome (complete or good pain relief vs partial or no pain relief), and rates of recurrence and complications including facial paralysis, weakness, or paresis; hearing loss; auditory and facial nerve damage; cerebrospinal fluid leakage; infection; cerebellar damage; and death.

Good pain relief was achieved in 81\% of MVD patients and 88\% of E-MVD patients, with a mean recurrence rate of $14 \%$ and $9 \%$, respectively. Average rates of reported complications were statistically lower in E-MVD than in MVD approaches, including facial paresis or weakness, hearing loss, cerebellar damage, infection, and death, whereas cerebrospinal fluid leakage was similar. The overall incidence of complications was 19\% for MVD and 8\% for E-MVD.

CONCLUSIONS The reviewed literature revealed similar clinical outcomes with respect to pain relief for MVD and EMVD. The recurrence rate was lower in E-MVD studies, though not significantly so, and the incidence of complications, notably facial paresis and hearing loss, were statistically higher for MVD than for E-MVD. Based on these results, the use of endoscopy to perform MVD for TN appears to offer at least as good a surgical outcome as the more commonly used open MVD, with the possible added advantages of having a shorter operative time, smaller craniotomy, and lower recurrence rates. The authors advise caution in interpreting these data given the asymmetry in the sample size between the two groups and the relative novelty of the E-MVD approach.

https://thejns.org/doi/abs/10.3171/2018.6.JNS172690

KEYWORDS trigeminal neuralgia; endoscopic microvascular decompression; functional neurosurgery; pain

ABBREVIATIONS CSF = cerebrospinal fluid; E-MVD = endoscopic MVD; MVD = microvascular decompression; TN = trigeminal neuralgia.

SUBMITTED October 27, 2017. ACCEPTED June 27, 2018.

INCLUDE WHEN CITING Published online December 7, 2018; DOI: 10.3171/2018.6.JNS172690. 
$\mathrm{M}$ ICROVASCULAR decompression (MVD) has become the accepted surgical technique for the treatment of trigeminal neuralgia (TN) because of its positive surgical outcomes and prevention of longterm recurrence. For some patients, pharmacological treatment is sufficient for adequate control of TN symptoms: severe, episodic facial pain caused by injury and inflammation of the trigeminal or fifth cranial nerve., ${ }^{8,12}$, 15,19,20,28 Ablative procedures have been deemed effective at improving acute TN symptoms, but MVD has demonstrated superiority in terms of short- and long-term pain relief outcomes and preventing long-term recurrence. ${ }^{3,13}$

Since MVD's inception, several important modifications-for example, patient positioning in the lateral position or park-bench position, nerve decompression rather than nerve section, and the use of intraoperative auditory brainstem evoked potentials to indicate early irritation of the cranial nerves and brainstem-have decreased surgical morbidity and improved patient outcomes. ${ }^{20,27}$ A reduction in the size of the microscope and refined instrumentation have resulted in a modern-day craniotomy and surgical corridor size that are comparable to those in endoscopic procedures, improving pain outcomes and lowering complication rates..$^{10,17}$

Positive outcome rates of MVD for TN are between $77 \%$ and $98 \%$, with the majority of patients pain free and asymptomatic at follow-up., ${ }^{9,11,22-26,30,31}$ However, a number of significant complications can develop due to retraction of the cerebellum and stretching of the vasa nervorum and other factors resulting in cerebrospinal fluid (CSF) leakage, facial paralysis, hearing loss, and sequelae of cerebellar damage. .11,18,26 Trigeminal neuralgia can also recur, particularly if a compressing vessel is overlooked during MVD. ${ }^{2,4}$

Fully endoscopic MVD (E-MVD) treatment for TN appears at first glance to be effective. ${ }^{1}$ Despite its minimally invasive nature, E-MVD appears to enable improved localization of neurovascular abnormalities, and decreased cerebellar retraction may prevent granuloma formation and adhesion at the decompression site, thereby promoting better surgical outcomes. ${ }^{7}$ Although craniotomy and surgical corridor diameters as well as surgical times for E-MVD procedures are similar to those for open MVD (approximately 1-cm incisions and 130-minute operational times for both), the smaller size of the endoscope leaves a greater space and allows for enhanced maneuverability of other surgical instruments within the operating field. ${ }^{10,17}$ However, the approach has gained momentum slowly. ${ }^{4,10}$ To date, there is no systematic literature review that compares open MVD and E-MVD outcomes in TN. Thus, we aimed to conduct an analysis of existing studies to compare patient outcome rates and complications between the two surgical procedures for the treatment of TN.

\section{Methods}

\section{Search Strategy and Selection Criteria}

The Preferred Reporting Items for Systematic Reviews and Meta-Analyses (PRISMA) methodology guidelines were followed for this meta-analysis. A search of the
PubMed/MEDLINE and Ovid databases was conducted for studies published from database inception to 2017. The search terms used included, but were not limited to, "open microvascular decompression," "microvascular decompression for trigeminal neuralgia," and "endoscopic decompression for trigeminal neuralgia." Relevant references from the chosen articles were also included.

Inclusion criteria for studies in the meta-analysis were established as follows: adult patients, clinical studies with $\geq 10$ patients (excluding case studies to obtain a higher volume of outcome rates), utilization of open MVD or E-MVD to treat TN, craniotomy and retrosigmoid incision, English-language studies, and articles that listed pain relief outcomes (complete, very good, partial, or absent), recurrence rate (number of patients), and complications (paresis, hearing loss, CSF leak, cerebellar damage, infection, death).

Exclusion criteria for the analysis were as follows: clinical studies involving other procedures (e.g., exoscopy, radiosurgery, balloon compression, glycerol rhizolysis, or percutaneous radiofrequency lesioning, CT); studies that did not address TN (e.g., nerve atrophy, brainstem trigeminal evoked potentials); pediatric, animal, basic science, and cadaver studies; comparative studies in which the extraction of accurate data was difficult; and commentary articles.

\section{Study Selection and Data Extraction}

Titles and abstracts were screened by two reviewers, who assessed full texts for adherence to the inclusion criteria listed above. Studies that did not fit the inclusion criteria, duplicates, and studies that fit the exclusion criteria outlined above were excluded. The two reviewers discussed any papers that were controversial, and a third reviewer assessed these papers in a blinded manner. Any disagreements about the inclusion or exclusion of a particular study were resolved by discussion or mediation by the two independent reviewers. Furthermore, the two independent reviewers looked at available abstracts and studies from non-English language publications and determined that they did not fit the inclusion criteria.

Data were collected according to the primary objective for the literature review, that is, to compare the difference in outcome rates between MVD and E-MVD approaches for patients with $\mathrm{TN}$. The null hypothesis proposed that there was no difference between the two surgical procedures in terms of pain relief (outcome 1) and recurrence (outcome 2) in the treatment of TN. Therefore, the percentage of patients who attained complete or good postoperative pain relief, partial relief, or no pain relief after MVD or E-MVD as well as the percentages of recurrence for both procedures was collected and analyzed.

The secondary objective was to compare rates of common complications following MVD versus E-MVD for treatment of TN. Therefore, data were collected on the incidence of facial paralysis, weakness, or paresis; hearing loss; auditory and facial nerve damage; CSF leakage; infection; cerebellar damage; and mortality following surgery. Additional outcomes such as hospital length of stay were also reviewed but not included in the analysis because of inconsistent reporting. Most studies did not 


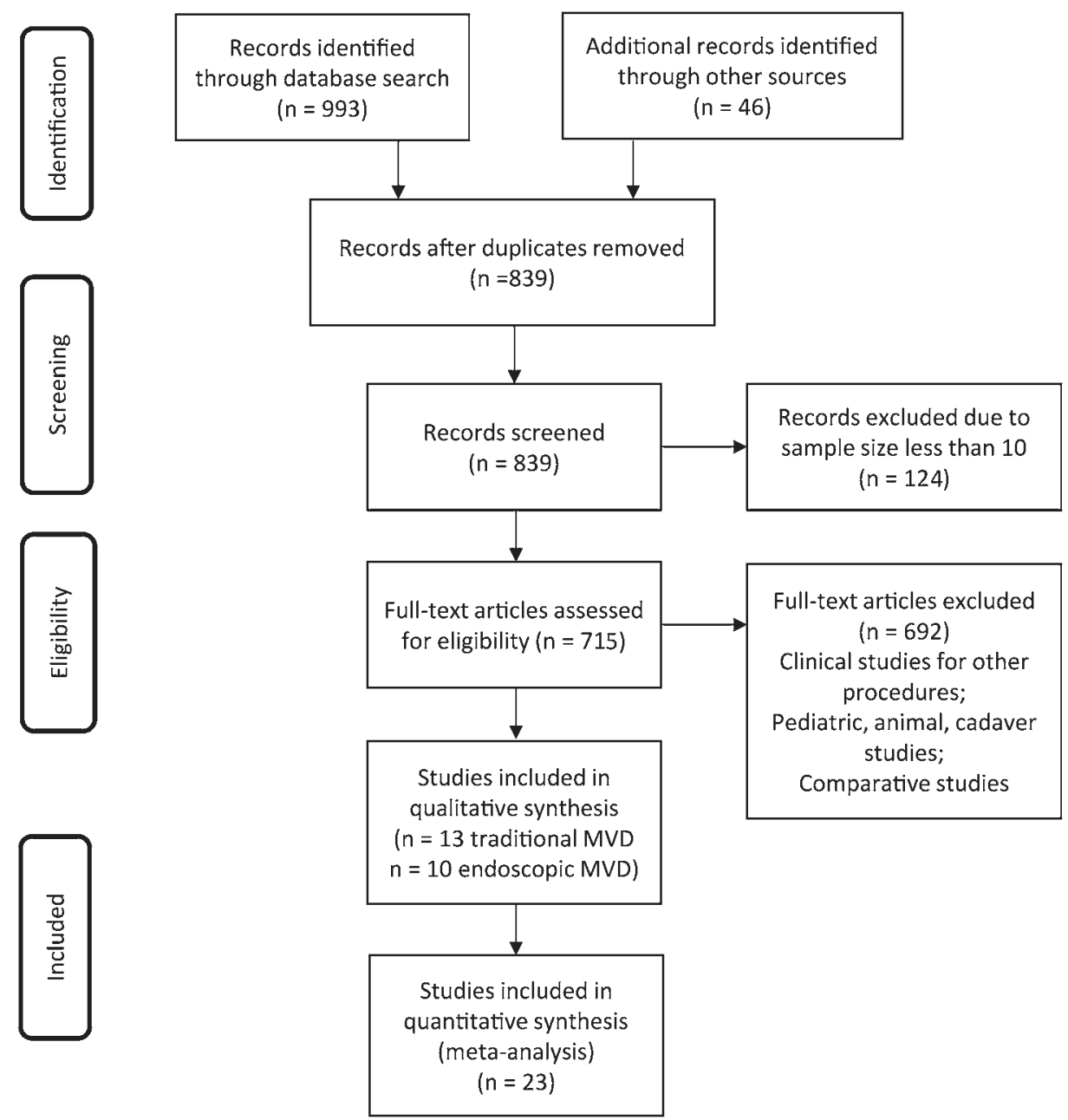

FIG. 1. Preferred Reporting Items for Systematic Reviews and Meta-Analyses (PRISMA) flowchart of the literature search and study selection.

include brainstem auditory evoked potentials, so we were unable to include this factor for a more accurate analysis of auditory function and hearing loss.

\section{Statistical Analysis}

A meta-analysis was performed by combining and comparing the reported incidence of pain relief, recurrence, and different complications for MVD versus EMVD for the treatment of TN. Proportion forest plots were charted to determine means, calculate $95 \%$ confidence intervals for all data, and compare the two study cohorts. StatsDirect 3 software (StatsDirect Ltd.) was used for the calculations. In this meta-analysis, results were plotted using the random effects model to take into account clinical diversity and methodological variation between studies.

One-way ANOVA was used to determine if outcomes including pain relief and surgical complications were equivalent between the open MVD and E-MVD studies. If the ANOVA failed to show significance, the variables were analyzed using a Wilcoxon rank-sum test.

Kaplan-Meier curves and probabilities were generated using log-rank survival analysis on SigmaPlot 12.0 soft- ware (Systat Software Inc.). Since most included studies were retrospective, precise times for follow-up and events of interest were not available. Thus, average follow-up times were used, along with the number of patients experiencing no or mild pain (censored) or moderate to severe pain (event) at follow-up. Only studies published later than the year 2000, the earliest publication year for the selected E-MVD studies, were included in this analysis in order to ensure an equivalent state of healthcare and medical technology between the two groups, as they are from the same time period, and to create a more balanced number of subjects. Note that 536 open MVD and 680 E-MVD patients were included as data points for generating the survival curves. The range of average follow-up times for each study allowed us to create a crude estimate of painfree survival times for MVD and E-MVD.

\section{Results}

From a larger pool of 1039 studies, we identified 839 unduplicated articles electronically and by manual search. Of these 839 articles, two independent reviewers eliminated 124 with a sample size smaller than 10 and another 692 
TABLE 1. Studies included in the meta-analysis

\begin{tabular}{|c|c|}
\hline Authors \& Year & No. of Patients \\
\hline \multicolumn{2}{|l|}{ Open MVD } \\
\hline Mendoza et al., 1995 & 133 \\
\hline Barker et al., 1996 & $1185^{*}$ \\
\hline McLaughlin et al., 1999 & 3196 \\
\hline Broggi et al., 2000 & 148 \\
\hline Olson et al., 2005 & 156 \\
\hline Pamir \& Peker, 2006 & 90 \\
\hline Forbes et al., 2011 & 59 \\
\hline Gao et al., 2017 & 52 \\
\hline Sandell \& Eide, 2008 & 135 \\
\hline Oesman \& Mooij, 2011 & 156 \\
\hline Bakker et al., 2014 & 33 \\
\hline Wei et al., 2018 & 425 \\
\hline Tomasello et al., 2016 & 15 \\
\hline \multicolumn{2}{|l|}{ E-MVD } \\
\hline El-Garem et al., 2002 & 42 \\
\hline Kabil et al., 2005 & 255 \\
\hline Teo et al., 2006 & 114 \\
\hline Artz et al., 2008 & 41 \\
\hline Chen et al., 2008 & 156 \\
\hline Yadav et al., 2011 & 51 \\
\hline Halpern et al., 2013 & 35 \\
\hline Bohman et al., 2014 & 47 \\
\hline Kher et al., 2017 & 178 \\
\hline Lee et al., 2017 & 74 \\
\hline
\end{tabular}

${ }^{*}$ Decompressions numbered 1204.

articles not relevant to our analysis. Twenty-three articles were ultimately selected for data extractable for the meta-analysis: 13 studies on traditional MVD and 10 on EMVD (Fig. 1). The total population included in the metaanalysis was 6749 patients: 5783 for the open MVD (5802 procedures) and 993 for the E-MVD approach (Table 1).

Clinical outcomes for both procedures (Table 2 and Figs. 2-5) were comparable, with an average of $81 \%(95 \%$ CI 74\%-86\%) complete or good postoperative pain relief with MVD and 88\% (95\% CI 83\%-93\%) with E-MVD. Mean recurrence was $14 \%(95 \%$ CI $8 \%-21 \%)$ for MVD and $9 \%(95 \%$ CI 5\%-14\%) for E-MVD. Good pain relief and pain recurrence were not statistically different between the open MVD and E-MVD studies.

Respective rates of complications after MVD versus EMVD were 9\% versus 3\% for facial paresis or weakness, $4 \%$ versus $1 \%$ for hearing loss, $2 \%$ versus $<1 \%$ cerebellar damage, $2 \%$ versus $<1 \%$ for infection, and $<1 \%$ versus $0 \%$ for deaths (Table 2 and Fig. 4). Complication rates were statistically lower after E-MVD than after open MVD for hearing loss $(p=0.0373)$, cerebellar damage $(p=0.0048)$, infection $(\mathrm{p}=0.0013)$, and death $(\mathrm{p}=0.0075)$, while there was only a trend for lower facial paresis rates after EMVD than after open MVD ( $p=0.0567)$. Cerebrospinal fluid leakage occurred in 3\% of MVD patients and 3\% of E-MVD patients. The overall incidence of complications
TABLE 2. Summary of meta-analysis comparing open MVD with E-MVD

\begin{tabular}{lcc}
\hline \multicolumn{1}{c}{ Outcome \& Complications } & $\begin{array}{c}\text { Open MVD, } \% \\
(95 \% \mathrm{Cl})\end{array}$ & $\begin{array}{c}\text { E-MVD, } \% \\
(95 \% \mathrm{Cl})\end{array}$ \\
\hline $\begin{array}{l}\text { Good pain relief outcome: com- } \\
\text { plete or very good vs partial } \\
\text { or absent }\end{array}$ & $81 \%(74 \%-86 \%)$ & $88 \%(83 \%-93 \%)$ \\
\hline Recurrence rate: major or minor & $14 \%(8 \%-21 \%)$ & $9 \%(5 \%-14 \%)$ \\
\hline Total complications after surgery & $19 \%(10 \%-30 \%)$ & $8 \%(5 \%-13 \%)$ \\
\hline Facial paresis or weakness & $9 \%(4 \%-16 \%)$ & $3 \%(0 \%-8 \%)$ \\
\hline Hearing loss & $4 \%(2 \%-6 \%)$ & $1 \%(0 \%-3 \%)$ \\
\hline CSF leakage & $3 \%(1 \%-6 \%)$ & $3 \%(2 \%-4 \%)$ \\
\hline Cerebellar damage & $2 \%(0 \%-4 \%)$ & $<1 \%(0 \%-0.2 \%)$ \\
\hline Infection & $2 \%(0 \%-3 \%)$ & $<1 \%(0 \%-0.2 \%)$ \\
\hline Death & $<1 \%(0 \%-2 \%)$ & $0 \%$ \\
\hline
\end{tabular}

was $19 \%(95 \%$ CI $10 \%-30 \%)$ for MVD and $8 \%(95 \%$ CI 5\%-13\%) for E-MVD approaches. The total incidence rate for surgical complications was significantly lower in the E-MVD studies than the open MVD studies ( $p=0.0374)$.

Pain-free survival following surgery was examined using Kaplan-Meier log-rank analysis (Fig. 6). Survival rates at 1,2, and 3 years were not drastically different between the two surgery types. Probabilities for pain-free survival following MVD and E-MVD, respectively, were 0.921 and 0.956 at 12 months, 0.911 and 0.943 at 24 months, and 0.896 and 0.903 at 36 months.

The range and total incidence of postoperative complications in each study varied significantly and may have been attributable to differences in, for example, length of postoperative follow-up, patient health and age, whether the surgery was primary or a revision, and length of time the patient had TN. Operator error is another variable difficult to adjust for.

\section{Discussion}

This literature review has revealed that E-MVD produces results that are comparable to those of the open technique, with lower rates of major or minor recurrence and statistically significant lower overall rates of complications following surgery, notably for cerebellar damage, infection, death, and hearing loss, with a near-significant trend for a lower rate of facial paresis or weakness. Rates of CSF leakage were at 3\% for both types of studies, and rates of cerebellar damage, infection, and death were comparable (all $\leq 2 \%$ for both approaches). These studies support the premise that the less invasive endoscopic approach is a favorable alternative to open MVD for the treatment of TN.

An important factor to keep in mind in any meta-analysis spanning several decades of research is that as surgical techniques become more common, technical and methodological improvements will impact outcome and complication rates. In a 1995 study of MVD, Mendoza et al. ${ }^{21}$ looked at 133 patients treated with posterior fossa MVD for TN between 1976 and 1992. Their findings showed that $71 \%$ of patients were pain free after the procedure, with $29 \%$ de- 
Good pain relief: open MVD

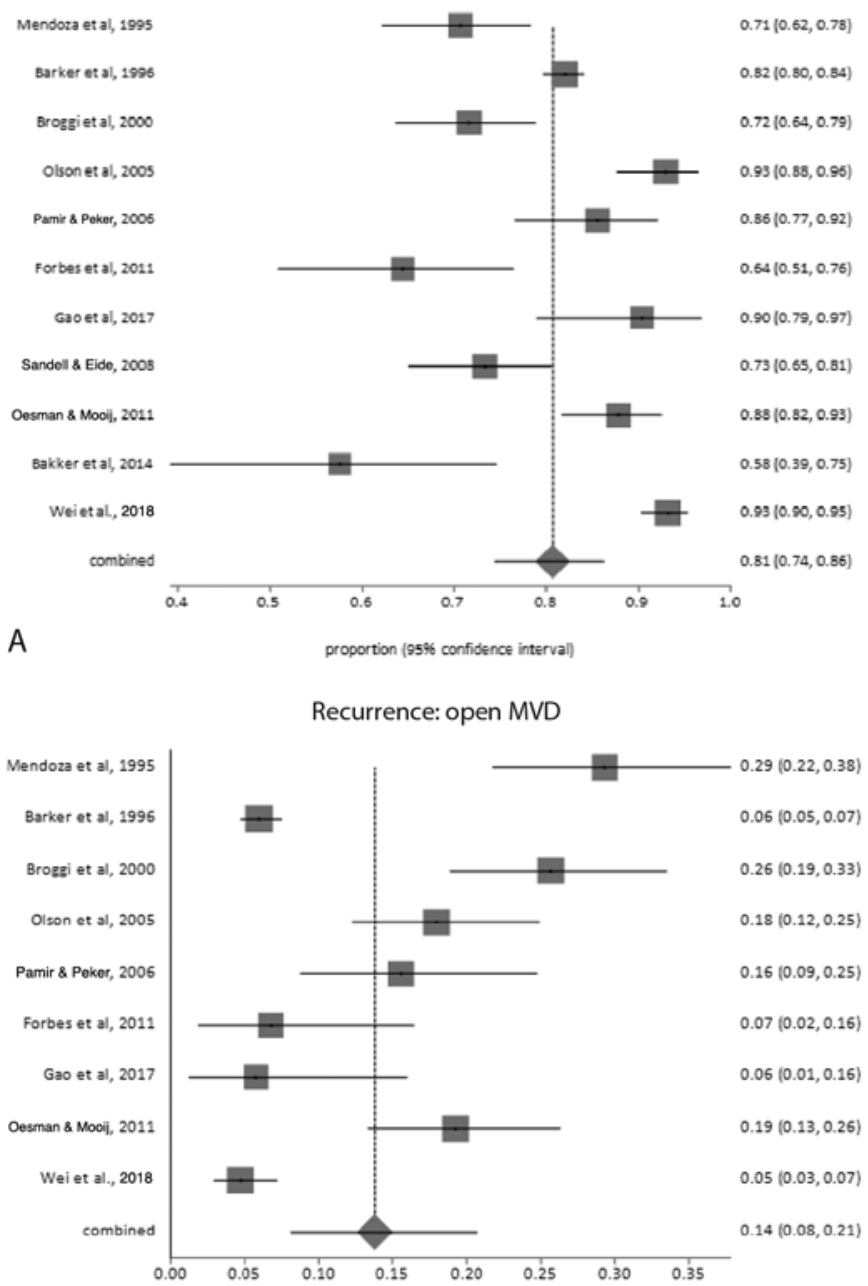

B

proportion (95\% confidence interval)

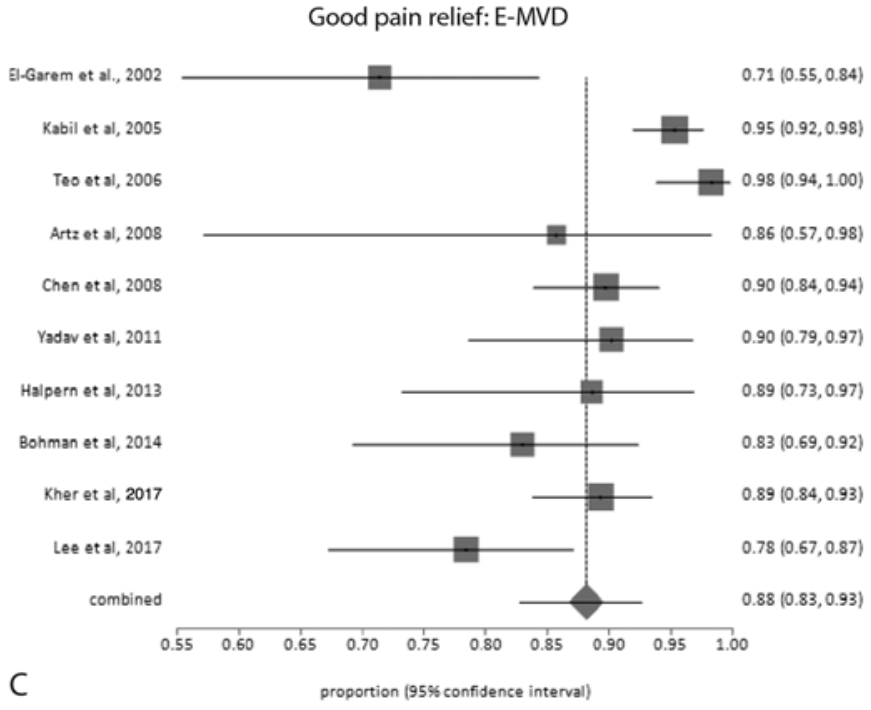

Recurrence: E-MVD

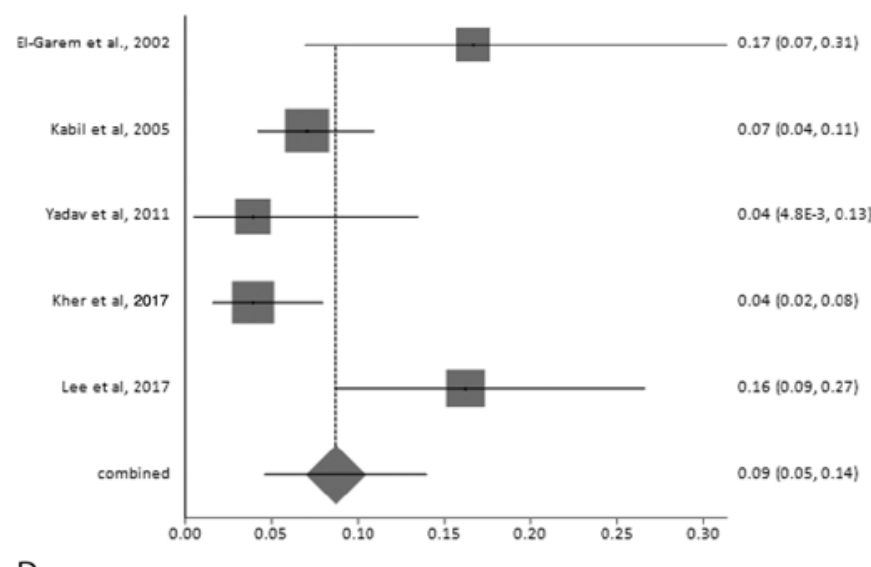

D

FIG. 2. Forest plots for surgical outcomes ( $95 \% \mathrm{Cl}$ includes continuity correction). A: Proportions of good pain relief outcomes (complete or very good) in the open MVD series (relevant data not included in McLaughlin et al., 1999, and Tomasello et al., 2016). B: Proportions of good pain relief outcomes (complete or very good) in the E-MVD series. C: Proportions of recurrences for the open MVD series (relevant data not included in McLaughlin et al., 1999; Sandell \& Eide, 2008; and Tomasello et al., 2016). D: Proportions of recurrences for the E-MVD series (relevant data not included in Teo et al., 2006; Artz et al., 2008; Chen et al., 2008; Halpern et al., 2013; and Bohman et al., 2014).

veloping recurrent TN. In 2000 Broggi et al. ${ }^{5}$ published a review of 148 patients with TN who had undergone MVD between 1990 and 1998, showing similar findings: 75\% postoperative relief. In 2017 Gao et al. reported a 90\% success rate, and in 2018 Wei et al. reported 93\% of patients who were pain free following surgery. ${ }^{9,31}$ Our sample size of 23 articles is not large enough to show a definite trend while controlling for variables such as length of follow-up. However, when comparing MVD and E-MVD outcomes, it is important to note that our study includes MVD articles dating from 1995 to 2017 and E-MVD articles dating between 2002 and 2017.

The endoscope has become a well-known tool in neurosurgery, but the transition from MVD to fully E-MVD has been slower and more recent. As E-MVD becomes more commonly used, improvements and applications may impact outcomes positively. Contrary to traditional MVD, fully E-MVD can be performed using 4-mm $0^{\circ}$ and $30^{\circ}$ endoscopes, where a pneumatic holding system is used to fix the endoscope, ${ }^{4,14}$ through which perforators and neurovascular compression can be detected. Transposition of the affected vessel is performed safely with fibrin glue and Teflon string, with preservation of the perforators. ${ }^{8}$

Using a traditional microscope during an operation allows 3D imaging and also has the advantage of being a familiar tool for most neurosurgeons. Using an endoscope provides 2D imaging (unless 3D scopes are used) but has the advantage of providing better visualization around corners than a microscope..$^{29}$ In addition, the surgeon can potentially work with a smaller surgical opening and avoid awkward positioning. Enhancing visualization via an endoscope is minimally invasive, enables the surgeon to identify the exact location of the neurovascular conflict, and provides a panoramic view with higher definition, bet- 


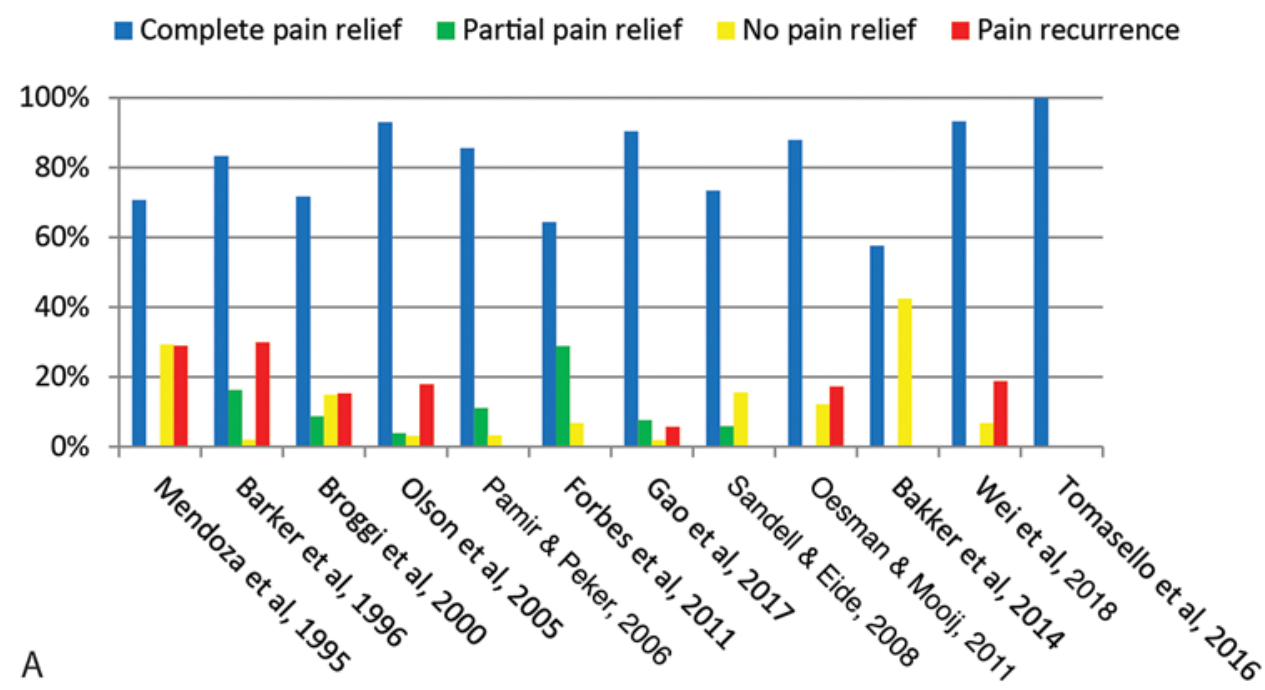

Proportion of pain outcomes by study: E-MVD

a Complete pain relief $\quad$ Partial pain relief $\quad$ No pain relief $\quad$ Pain recurrence

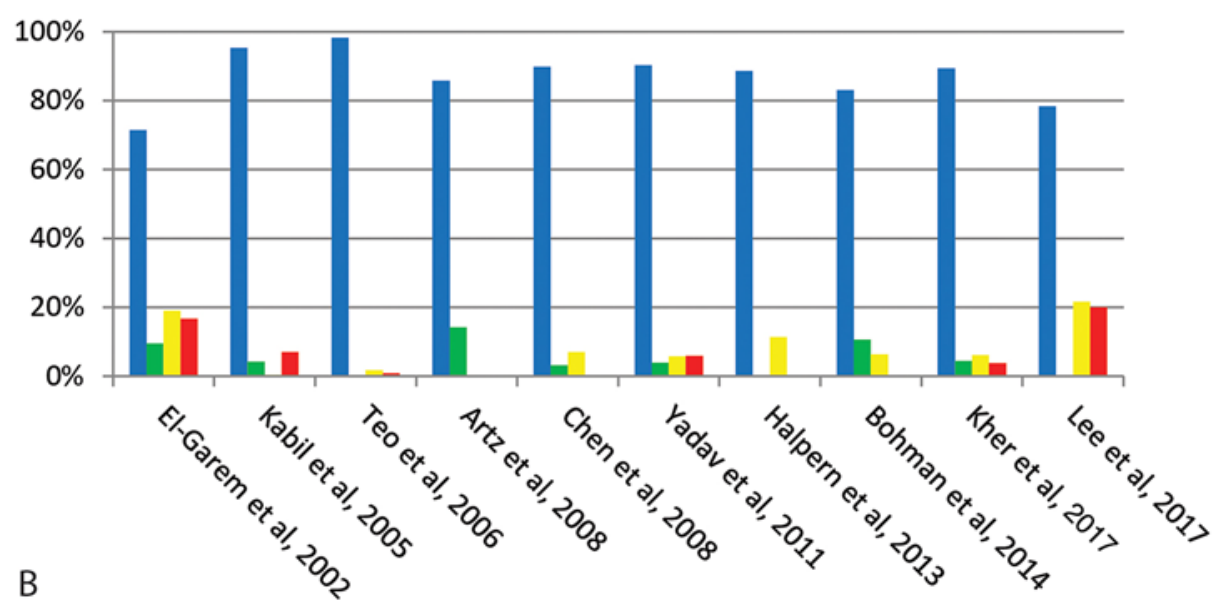

FIG. 3. Percentages of pain relief outcomes and recurrence rates in open MVD studies (relevant data not included in McLaughlin et al., 1999; A) and E-MVD studies (B). Figure is available in color online only.

ter illumination, and more flexibility than the traditional approach. ${ }^{16,32}$ Angled endoscopes provide further visualization advantages during surgery.

However, it is difficult to easily use endoscopes without training and practice. Angled scopes are particularly difficult to work with and can only be used with angled instruments, unless visualization alone is the goal. The learning curve in becoming adept at using an endoscope during surgery for MVD varies. At a minimum, however, repetitive practice with endoscopes is needed for ease of use. An excellent camera operator is also a prerequisite for successfully conducting E-MVD. Scope holders can be used instead of a camera operator, but most surgeons prefer the dynamic range of movement an operator can provide, rather than a static scope holder.

Transition to E-MVD is advantageous, as several studies have demonstrated clinical improvement in the absence of permanent complications in many cases., ${ }^{4,717}$ In 2002 ElGarem et al. ${ }^{7}$ reported that the use of endoscopy to perform MVD of the trigeminal nerve for 42 patients resulted in complete pain relief for 30 patients $(71 \%)$ and partial pain relief for 4 patients (10\%). These percentages are comparable to the clinical outcomes for MVD. Studies performed 10-15 years after El-Garem's documented between $78 \%$ and $95 \%$ favorable outcomes.

Overall, similar clinical outcomes and incidences of postoperative complications were reported for the MVD and E-MVD studies. The incidence of postoperative complications, particularly facial paresis or weakness and hearing loss, was lower in the E-MVD studies than in the MVD studies. However, given the considerable variability in conditions between studies, a direct comparison is difficult. For example, according to one study, cases of cerebral hemorrhage or CSF leakage could be attributed to op- 

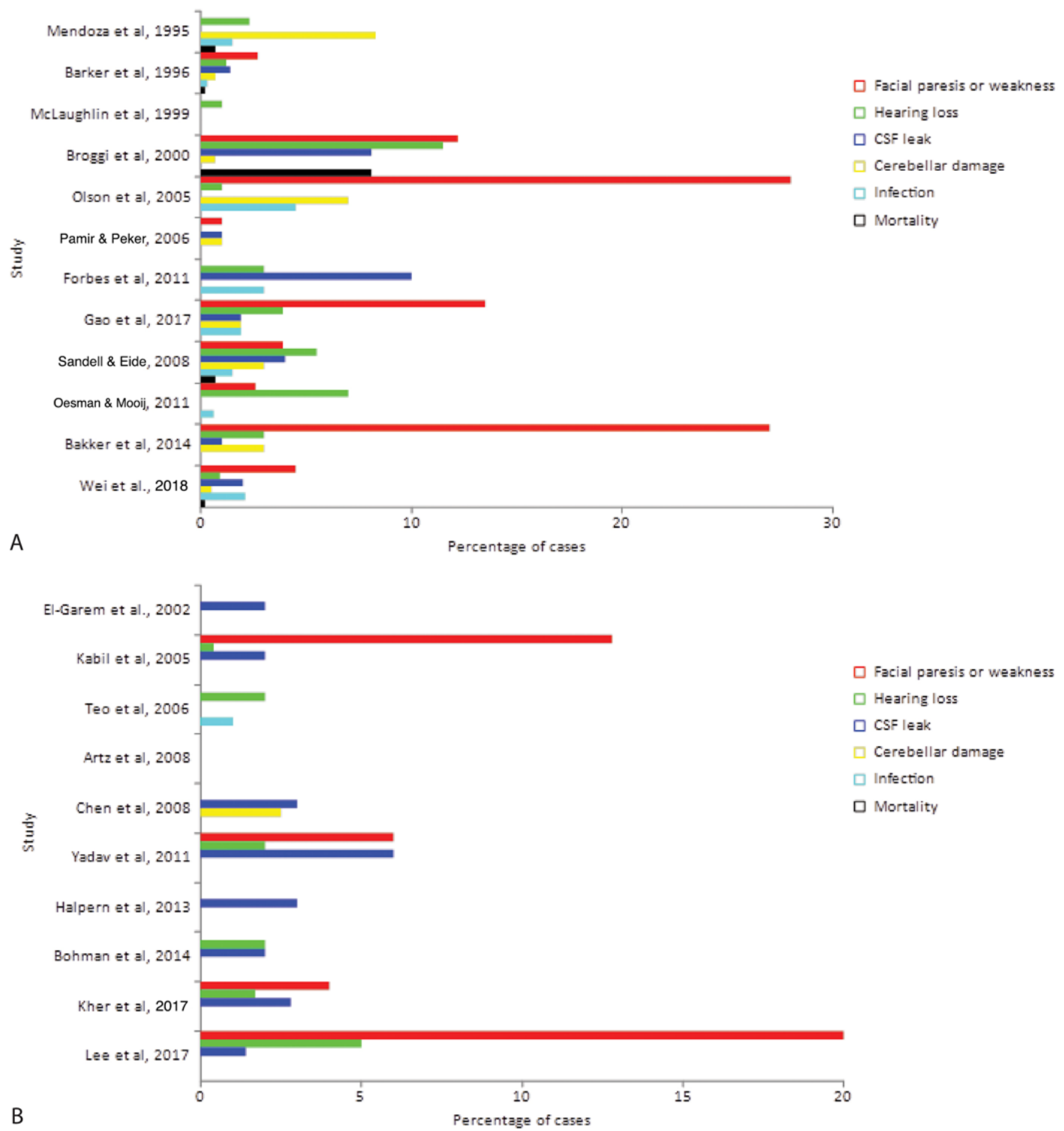

FIG. 4. Percentage of cases of the six most common complications following open MVD (A) and E-MVD (B) for TN. Figure is available in color online only.

erator error. ${ }^{6}$ In such instances, the authors recommended that proper training be undertaken in order to avoid unnecessary injury to patients. ${ }^{6}$ A qualitative measurement of neurological function and damage, such as brainstem auditory evoked potentials, would be useful for a more accurate comparison, but most of the studies reviewed did not include such an analysis. Notably, no patient deaths were reported for the E-MVD studies; however, sample size was smaller for those studies than for MVD.

Data on length of stay were not consistently extractable from the studies to make a valid comparison. In addition, reporting of the recurrence rate was not consistent for the MVD or E-MVD studies, thereby limiting comparison of this outcome. This could be attributable to poor 

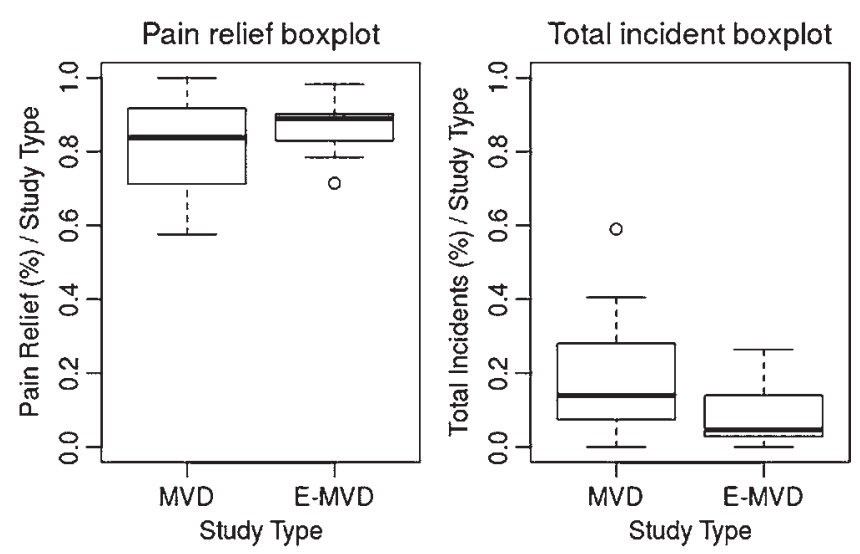

FIG. 5. Box plots showing the distribution of good pain relief outcome percentages (left) and recurrence rates (right) among the open MVD and E-MVD studies.

and inconsistent follow-up visits, especially for long-term studies. We acknowledge the following limitations in the study: the significant heterogeneity between the studies and poor availability of comparable data; the crossover of patient populations between a number of studies; different definitions of measurements, especially for pain relief and recurrence rate; and significant differences in population numbers and group sizes.

\section{Conclusions}

The outcome rates and incidence of postoperative complications following E-MVD versus MVD in our study provide supportive evidence that performing fully E-MVD affords patients clinical results comparable with those of an open MVD approach but with lower complication rates,

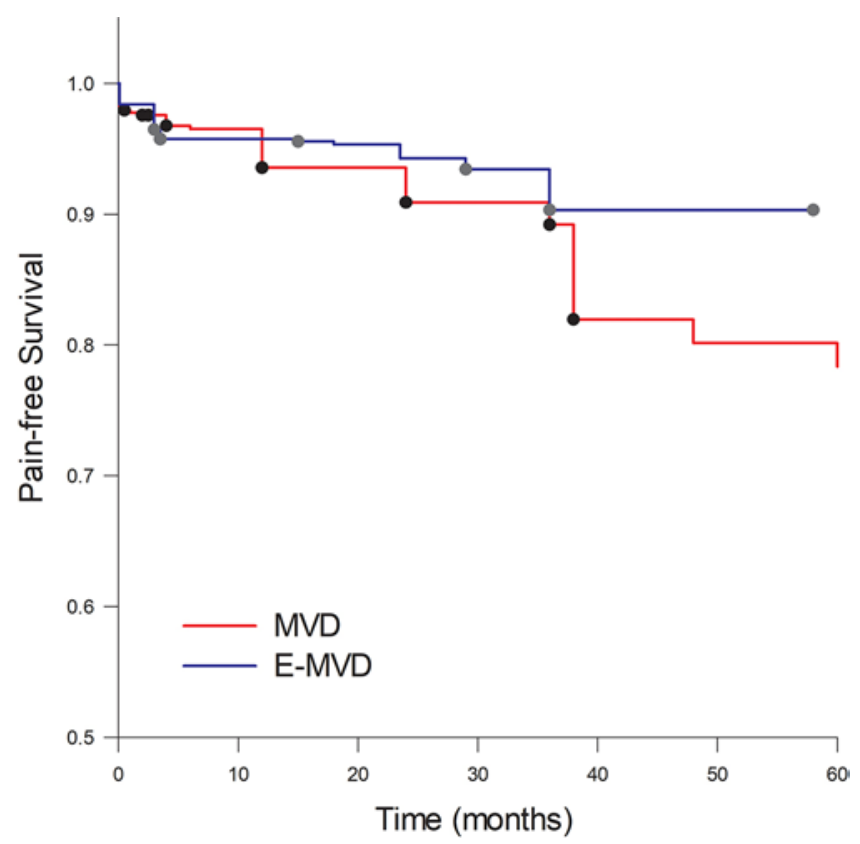

FIG. 6. Kaplan-Meier pain-free survival curves following open MVD and E-MVD surgery. Mean follow-up times from each applicable study were used, with an event being the recurrence of moderate to severe pain. Figure is available in color online only. notably facial paresis or weakness and hearing loss, following surgery for TN. However, caution is recommended in the interpretation of our results because of unquantifiable factors such as an unknown level of surgical training or operator errors. Future studies should further strengthen the conclusions of this review and meta-analysis. Nonetheless, the superb illumination and visualization of perforators arising from the offending structure (e.g., artery, vessel) contributing to the TN symptoms is an added benefit to the surgeon. Additional long-term studies with larger samples and consistent parameters and controls are needed to further compare E-MVD to traditional MVD.

\section{References}

1. Artz GJ, Hux FJ, Larouere MJ, Bojrab DI, Babu S, Pieper DR: Endoscopic vascular decompression. Otol Neurotol 29:995-1000, 2008

2. Bakker NA, Van Dijk JM, Immenga S, Wagemakers M, Metzemaekers JD: Repeat microvascular decompression for recurrent idiopathic trigeminal neuralgia. J Neurosurg 121:936-939, 2014

3. Barker FG II, Jannetta PJ, Bissonette DJ, Larkins MV, Jho HD: The long-term outcome of microvascular decompression for trigeminal neuralgia. N Engl J Med 334:1077-1083, 1996

4. Bohman LE, Pierce J, Stephen JH, Sandhu S, Lee JY: Fully endoscopic microvascular decompression for trigeminal neuralgia: technique review and early outcomes. Neurosurg Focus 37(4):E18, 2014

5. Broggi G, Ferroli P, Franzini A, Servello D, Dones I: Microvascular decompression for trigeminal neuralgia: comments on a series of 250 cases, including 10 patients with multiple sclerosis. J Neurol Neurosurg Psychiatry 68:59-64, 2000

6. Chen MJ, Zhang WJ, Yang C, Wu YQ, Zhang ZY, Wang Y: Endoscopic neurovascular perspective in microvascular decompression of trigeminal neuralgia. J Craniomaxillofac Surg 36:456-461, 2008

7. El-Garem HF, Badr-El-Dine M, Talaat AM, Magnan J: Endoscopy as a tool in minimally invasive trigeminal neuralgia surgery. Otol Neurotol 23:132-135, 2002

8. Forbes J, Cooper C, Jermakowicz W, Neimat J, Konrad P: Microvascular decompression: salient surgical principles and technical nuances. J Vis $\operatorname{Exp}(\mathbf{5 3}):$ e2590, 2011

9. Gao J, Fu Y, Guo SK, Li B, Xu ZX: Efficacy and prognostic value of partial sensory rhizotomy and microvascular decompression for primary trigeminal neuralgia: a comparative study. Med Sci Monit 23:2284-2291, 2017

10. Halpern CH, Lang SS, Lee JY: Fully endoscopic microvascular decompression: our early experience. Minim Invasive Surg 2013:739432, 2013

11. Hitotsumatsu T, Matsushima T, Inoue T: Microvascular decompression for treatment of trigeminal neuralgia, hemifacial spasm, and glossopharyngeal neuralgia: three surgical approach variations: technical note. Neurosurgery 53:14361443,2003

12. Jannetta PJ: Arterial compression of the trigeminal nerve at the pons in patients with trigeminal neuralgia. J Neurosurg 26 (1 Suppl):159-162, 1967

13. Kabatas S, Albayrak SB, Cansever T, Hepgul KT: Microvascular decompression as a surgical management for trigeminal neuralgia: a critical review of the literature. Neurol India 57:134-138, 2009

14. Kabil MS, Eby JB, Shahinian HK: Endoscopic vascular decompression versus microvascular decompression of the trigeminal nerve. Minim Invasive Neurosurg 48:207-212, 2005

15. Katusic S, Beard CM, Bergstralh E, Kurland LT: Incidence 
and clinical features of trigeminal neuralgia, Rochester, Minnesota, 1945-1984. Ann Neurol 27:89-95, 1990

16. Kher Y, Yadav N, Yadav YR, Parihar V, Ratre S, Bajaj J: Endoscopic vascular decompression in trigeminal neuralgia. Turk Neurosurg 27:998-1006, 2017

17. Lee JYK, Pierce JT, Sandhu SK, Petrov D, Yang AI: Endoscopic versus microscopic microvascular decompression for trigeminal neuralgia: equivalent pain outcomes with possibly decreased postoperative headache after endoscopic surgery. J Neurosurg 126:1676-1684, 2017

18. Liu J, Yuan Y, Fang Y, Zhang L, Xu XL, Liu HJ, et al: Microvascular decompression for atypical hemifacial spasm: lessons learned from a retrospective study of 12 cases. J Neurosurg 124:397-402, 2016

19. Love S, Coakham HB: Trigeminal neuralgia: pathology and pathogenesis. Brain 124:2347-2360, 2001

20. McLaughlin MR, Jannetta PJ, Clyde BL, Subach BR, Comey $\mathrm{CH}$, Resnick DK: Microvascular decompression of cranial nerves: lessons learned after 4400 operations. J Neurosurg 90:1-8, 1999

21. Mendoza N, Illingworth RD: Trigeminal neuralgia treated by microvascular decompression: a long-term follow-up study. Br J Neurosurg 9:13-19, 1995

22. Miller JP, Magill ST, Acar F, Burchiel KJ: Predictors of longterm success after microvascular decompression for trigeminal neuralgia. J Neurosurg 110:620-626, 2009

23. Oesman C, Mooij JJ: Long-term follow-up of microvascular decompression for trigeminal neuralgia. Skull Base 21:313322,2011

24. Olson S, Atkinson L, Weidmann M: Microvascular decompression for trigeminal neuralgia: recurrences and complications. J Clin Neurosci 12:787-789, 2005

25. Pamir MN, Peker S: Microvascular decompression for trigeminal neuralgia: a long-term follow-up study. Minim Invasive Neurosurg 49:342-346, 2006

26. Sandell T, Eide PK: Effect of microvascular decompression in trigeminal neuralgia patients with or without constant pain. Neurosurgery 63:93-100, 2008

27. Shin JC, Kim YC, Park CI, Chung UH: Intraoperative moni- toring of microvascular decompression in hemifacial spasm. Yonsei Med J 37:209-213, 1996

28. Simmons JH, Prehn RS: Nocturnal bruxism as a protective mechanism against obstructive breathing during sleep. Sleep 31:A199, 2008 (Poster)

29. Teo C, Nakaji P, Mobbs RJ: Endoscope-assisted microvascular decompression for trigeminal neuralgia: technical case report. Neurosurgery 59 (4 Suppl 2):E489-E490, 2006

30. Tomasello F, Esposito F, Abbritti RV, Angileri FF, Conti A, Cardali SM, et al: Microvascular decompression for trigeminal neuralgia: technical refinement for complication avoidance. World Neurosurg 94:26-31, 2016

31. Wei Y, Pu C, Li N, Cai Y, Shang H, Zhao W: Long-term therapeutic effect of microvascular decompression for trigeminal neuralgia: Kaplan-Meier analysis in a consecutive series of 425 patients. Turk Neurosurg 28:88-93, 2018

32. Yadav YR, Parihar V, Agarwal M, Sherekar S, Bhatele P: Endoscopic vascular decompression of the trigeminal nerve. Minim Invasive Neurosurg 54:110-114, 2011

\section{Disclosures}

The authors report no conflict of interest concerning the materials or methods used in this study or the findings specified in this paper.

\section{Author Contributions}

Conception and design: all authors. Acquisition of data: all authors. Analysis and interpretation of data: all authors. Drafting the article: Zagzoog. Critically revising the article: Zagzoog. Reviewed submitted version of manuscript: Zagzoog. Approved the final version of the manuscript on behalf of all authors: Zagzoog.

\section{Correspondence}

Nirmeen Zagzoog: Hamilton General Hospital, Hamilton, ON, Canada.nirmeen.zagzoog@medportal.ca. 